\title{
Infrared Spectroscopy of Cosmetic Gels Based on Medicinal Plants
}

\author{
Mosyagin V.V. ${ }^{1, *}$ Ryzhkova G.F. ${ }^{1}$ Bialyaev A.G. ${ }^{2}$ Minenkov N.A. ${ }^{1}$ Kanunnikova
}

T.V. ${ }^{1}$ Lebedeva N.V. ${ }^{1}$

${ }^{1}$ Kursk State Agricultural Academy named after I.I. Ivanov, 70, Karl Marx Str., Kursk, 305021, Russia

${ }^{2}$ Southwest State University, 94, 50 October St., Kursk, 305040, Russia

*Corresponding author. Email: ugnoe_nebo@list.ru

\begin{abstract}
Medicinal plants used in cosmetology are subject to mandatory quality control. For this purpose, various physical and chemical methods of study are most widely used. Infrared spectroscopy for the first time conducted a comparative study of cosmetic gels based on mint infusions (Mentha piperita), thyme (Thymus serpyllum) and celandine (Chelidonii Herba). The results of medium-wave spectroscopy revealed peaks of infrared absorption in different wavelength ranges for cosmetic gels obtained on the basis of infusions of different medicinal plants. Thus, for a gel based on thyme infusion they amounted to 2900.41; 2830.12; $2123.51 ; 1223.12 \mathrm{~cm}^{-1}$; celandine - 3527.17; 2879.64; 2822.89; 1449.25; 1393.66; 1290.91; 1116.51; $1083.03 \mathrm{~cm}^{-1}$; mint $-3863.60 ; 3724.47 ; 2904.68 ; 2835.10 ; 1453.00 ; 1271.44 ; 1111.11 ; 1102.16 ; 1034.30 \mathrm{~cm}^{-1}$. Within the infrared spectrum of cosmetic gels the obtained data allowed identifying the stretching band of carbonyl, hydroxyl, carboxyl, methyl, ether, acetal groups, compounds having aromatic ring, heterocyclic rings, in particular pyridine and quinolinic rings; mineral substances containing mainly phosphorus and sulfur compounds These groups and compounds are typical for some chemical compounds having biological effects (terpenoids, carotenoids, alkaloids, flavonoids, vitamins, acetylsalicylic, ursolic, oleanolic and other organic acids and compounds). The results of the study can be used as reference identification IR spectra for rapid identification of cosmetic gels based on infusions of medicinal plants, while the use of Fourier IR spectroscopy for studying the chemical composition of cosmetic gels based on infusions of medicinal plants can be of wide practical importance as a method of rapid analysis of cosmetic gels.
\end{abstract}

Keywords: cosmetology, medicinal plants, medium-wave spectroscopy

\section{INTRODUCTION}

Infrared spectroscopy is now widely used in biology. The IR spectrum analysis allows determining physical-chemical or biological characteristics of a sample, for example, chemical composition, granule size, density, etc. [5]. At present, there are databases of infrared spectra of food products, technical and food additives, medicines, poly- and monomers, plasticizers, toxic chemicals, solvents, petroleum products, toxic substances, steroids and other compounds having mainly plant-specific single-component composition [2, 8]. However, there is no reliable, complete and accessible base of infrared spectra of medicinal plant raw materials and thus based cosmetic preparations having a multicomponent composition [7, 9]. In order to identify various active components of medicinal plants transferred into infusion, it is advisable to use infrared spectroscopy with Fourier transform [1,6]. This method of analysis of cosmetic gels based on infusions of medicinal plants is not well studied, only the results of analysis of medicinal plants are given in the available works $[3,4]$. Since the chemical composition of medicinal plants is diverse, it is often impossible to use near-wave infrared spectroscopy in the analysis of plant raw materials [1].

\section{PURPOSE OF THE STUDY}

The purpose of the study is to analyze the possibility of using medium-wave infrared spectroscopy to identify cosmetic gels based on infusions of medicinal plants compared to control samples (aqueous gel Carbomer-940).

\section{OBJECT OF THE STUDY}

The object of the study included samples of cosmetic gels based on infusions of medicinal plants and gel based on Carbomer-940. 


\section{MATERIALS AND METHODS}

\subsection{Production of experimental cosmetic gels}

The samples of cosmetic gels were made from medicinal plants: peppermint (Mentha piperita), thyme (Thymus serpyllum) by LLC Pharmgroup, celandine (Chelidonii Herba) by LLC Health company. Medicinal plants were ground to particles of not more than $5 \mathrm{~mm}$, then the sample of medicinal herb was placed in a perforated infused glass, and then in an infused cup heated in advance in a boiling water bath for 15 minutes, poured with room temperature water, covered with a lid and infused in a boiling water bath. The infused cup was then removed from the water bath, kept for 45 minutes at room temperature, after which it was strained, the rest of the medicinal plant stock was squeezed off and boiled distilled water was added to a volume of $100 \mathrm{ml}$
(OFC.1.4.1.0018.15.). As a gelling component we used $0.1 \%$ Carbomer-940 manufactured in the United States.

\subsection{Study of IR spectra of cosmetic gels based on infusions of medicinal herbs}

The study of IR spectra of cosmetic gels based on medicinal plants was conducted on the Nicolet iS10 IRFourier spectrometer (Thermo Fisher Scientific).

\section{RESULTS AND DISCUSSION}

Figure 1 shows the results of a comparative study of IR spectra of cosmetic gels based on infusions of medicinal plants and Carbomer-940.

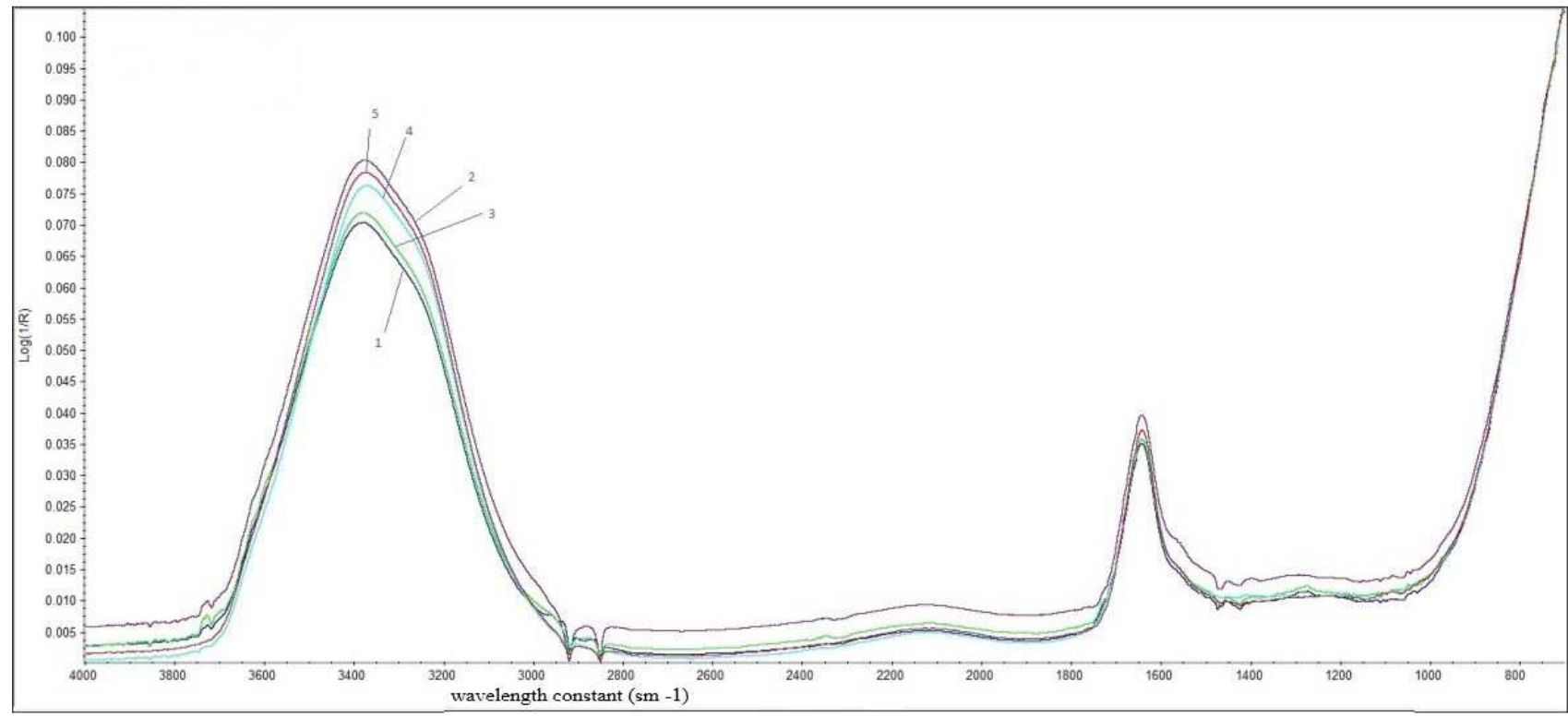

Figure 1. IR spectra of cosmetic gels based on infusions of medicinal plants and Carbomer-940. Main components: 1 gel sample with thyme infusion; 2 - gel sample with celandine infusion; 3 - gel sample with mint infusion; 4 - gel sample with sage infusion; 5 - Carbomer-940

Cosmetic gels based on infusions of medicinal plants (Figure 1, curves 1, 2, 3,5) contain specific components absorbing IR in areas that do not coincide with the radiation peaks absorbed by Carbomer-940. The nature of intensity of absorption bands of cosmetic gels based on medicinal plants can be inferred by their enlarged fragments shown in Figures 2-5.

The IR spectrum of cosmetic gel based on thyme infusion (Figure 2) shows peaks with the maxima of 2900.41; 2830.12; 2123.51; $1223.12 \mathrm{~cm}^{-1}$.

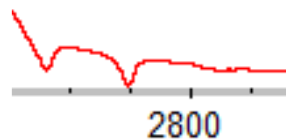

(a)

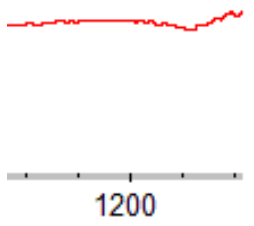

(b) 
Figure 2 Fragments of IR spectrum of cosmetic gel based on thyme infusion shown in Figure 1: a - peaks with the maxima of $2900.41 ; 2830.12 \mathrm{~cm}^{-1}$; $\mathrm{b}$ - peak with the maxima $1223.12 \mathrm{~cm}^{-1}$

This indicates the presence of hydroxyl groups and minerals in the gel containing mainly phosphorus and sulfur compounds. Fragments of IR spectrum of cosmetic gel based on celandine infusion shown in Figure 1.
The IR spectrum of cosmetic gel based on celandine infusion (Figure 3) shows peaks with the maxima of $3527.17 ; 2879.64 ; 2822.89 ; 1449.25 ; 1393.66 ; 1290.91$; 1116.51 1083.03

$\mathrm{cm}^{-1}$.
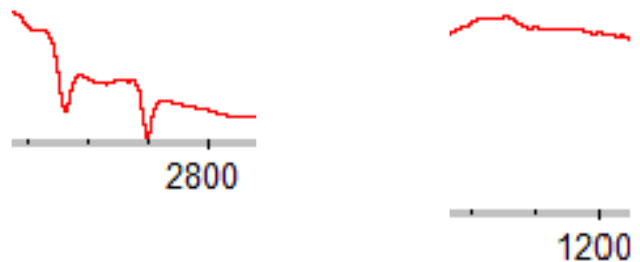

(a)

(b)

Figure 3 a - peaks with the maxima of $3527.17 ; 2879.64 ; 2822.89 ; \mathrm{cm}^{-1} ; \mathrm{b}$ - peak with the maxima 1449.25; 1393.66; $1290.91 ; 1116.51 ; 1083.03 \mathrm{~cm}^{-1}$

This indicates the presence of carbonyl, hydroxyl, carboxyl, methyl groups in the gel; aromatic ring compounds, particularly aromatic acid esters, phenols; heterocyclic rings, particularly pyridinic and quinolinic rings.
The IR spectrum of cosmetic gel based on mint infusion (Figure 4) shows peaks with the maxima of 3863.60; 3724.47 ; 2904.68; 2835.10; 1453.00; 1271.44; 1111.11; $1102.16 ; 1034.30 \mathrm{~cm}^{-1}$, thus indicating the presence of hydroxyl, carbonyl, ether, acetal groups in the gel.

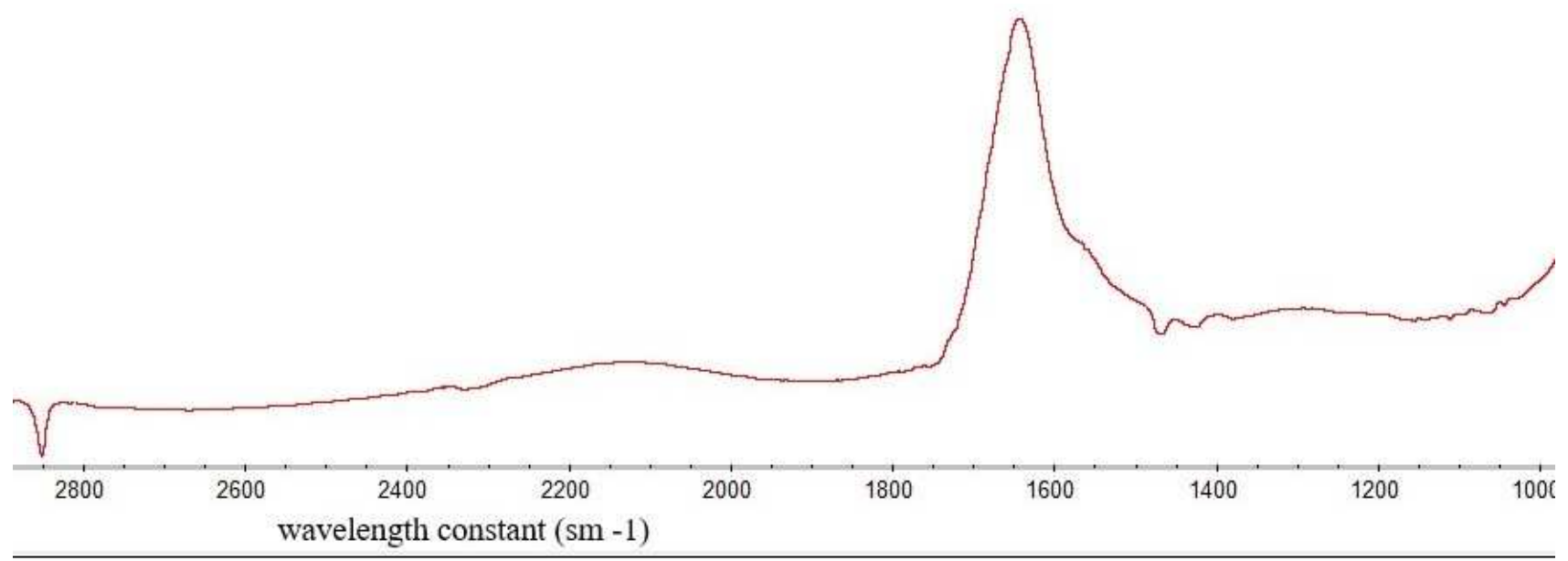

Figure 4 Fragments of IR spectrum of cosmetic gel based on shown in Figure 1

The IR spectrum of cosmetic gel based on sage infusion (Fig. 5) shows peaks with the maxima of 3902.20; 3863.33; 3863.44; 3840.60; 3819.69; $3719.09 \mathrm{~cm}^{-1}$, which could not be identified according to literary sourses.

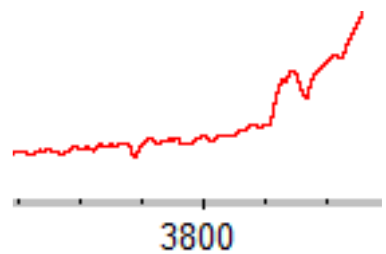

Figure 5 Fragments of IR spectra of cosmetic gel based on sage shown in Figure 1 
The analysis of literature sources shows [3] that the chemical composition of the medicinal plants used for the preparation of cosmetic gels contains a wider set of chemicals containing different functional groups than those obtained in laboratory studies using IR-Fourier spectrometer.

\section{CONCLUSION}

IR spectra of cosmetic gels based on medicinal plants thyme, celandine, mint and sage - were studied in comparison with Carbomer-940 used as a control sample. The obtained results show the presence of different functional groups in gels based on medicinal herbs, which are typical for some chemical compounds having biological effects (terpenoids, carotenoids, alkaloids, flavonoids, vitamins, minerals, ascorbic, ursolic, oleanolic and other organic acids and compounds), which is consistent with literary sources on the chemical composition of these medicinal plants.

\section{REFERENCES}

[1] A.P. Arzamastsev, N.P. Sadchikova, A.V. Titova, Method of near infrared spectroscopy in the system of drug quality control (review), Issues of boil., med. and pharmac. Chem. 1 (2010) 16-20.

[2] D.F. Valiulina, N.V. Makarova, D.V. Budylin, Comparative analysis of the chemical composition and antioxidant properties of different types of tea as a raw material for the production of tea extracts, Proc. of Voronezh State Univer. of Engineer. Technol. 80(2) (2018) 249-255.

[3] V.P. Moiseev, Physiology and biochemistry of plants. Methodological guidelines, 2rd ed., updated and revised, Belarusian State Agricult. Acad., 2009.

[4] L.P. Nilova, R.A. Ikramov, S.M. Malyutenkova, A.S. Veryaskina, Investigation of mineral composition during processing of wild berries, Proc. of Voronezh State Univer. of Engineer. Technol. 80(1) (2018) 151-156.

[5] A.V. Siedin, T.V. Orlovskaya, M.V. Gavrilin, Use of IR spectroscopy for rapid identification of glycosides in plant raw materials, Modern probl. of sci. and ed. 1 (2014) 367.

[6] B.N. Tarasevich, Fundamentals of IR spectroscopy with Fourier transform. Preparation of samples in IR spectroscopy, Moscow State Univer., Moscow, 2012.

[7] B.N. Tarasevich, IR spectra of basic classes of organic compounds. Reference materials, Moscow State Univer., Moscow, 2012.

[8] O.V. Chernousova, A.I. Krivtsova, T.A. Kuchmenko, The study of antioxidant activity of white tea, Proc. of Voronezh State Univer. of Engineer. Technol. 80(1) 2018 133-139.

[9] E.I. Ernandes, Y.A. Yuzkovskaya, New cosmetology, Fundamentals of modern cosmetology, LLC ID Cosmetics and Med., Moscow, 2019. 